

\title{
Rank tests for nonlinear
}

\author{
cointegration
}

\author{
Jörg Breitung \\ Humboldt-Universität zu Berlin \\ Institut für Statistik und Ökonometrie \\ Spandauer Strasse 1 \\ D-10178 Berlin
}

\begin{abstract}
A test procedure based on ranks is suggested to test for nonlinear cointegration. For two (or more) time series it is assumed that there exist monotonic transformations such that the normalised series can asymptotically be represented by independent Brownian motions. Rank test procedures based on the difference between the sequences of ranks are suggested. If there is no cointegration between the time series, the sequences of ranks tend to diverge, whereas under cointegration the sequences of ranks evolve similarly. Monte Carlo simulations suggest that for a wide range of nonlinear models the rank tests perform better than their parametric competitors. To test for nonlinear cointegration a variable addition test based on ranks is suggested. As empirical illustrations we consider the term structure of interest rates. Only weak evidence for a nonlinear long run relationship between interest yields of bonds with different time to maturity is found.
\end{abstract}

KEYWORDS: nonlinear cointegration, rank test, unit roots, arcsine distribution.

*I am indebted to Rolf Tschernig and Halbert White for helpful comments and suggestions. Of course, I am responsible for all remaining errors and deficiencies. Furthermore, I gratefully acknowledge financial support from the Sonderforschungsbereich 373 at the Humboldt University Berlin. 


\section{Introduction}

Since the introduction of the concept of cointegration by Granger (1981) the analysis of cointegrated models was intensively studied in a linear context, whereas the work on the extension to nonlinear cointegration is still comparatively limited. Useful reviews of recent work in the analysis of nonlinear cointegration are provided by Granger and Teräsvirta (1993), Granger (1995), and Granger, Inoue and Morin (1997).

In many cases, economic theory suggests a nonlinear relationship as for the production function or the Phillips curve, for example. However, theory does not always provide a precise specification of the functional form so that it might be desirable to have nonparametric tools for estimation and inference. In this paper rank test procedures are considered to test whether there is a possible nonlinear cointegration relation among the variables and whether this relationship is nonlinear.

To illustrate the problems of nonlinear cointegration it is helpful to consider a simple example. Let $x_{t}$ be nonlinear random walk given by $x_{t}^{3}=\sum_{j=1}^{t} v_{j}$, where $\left\{v_{t}\right\}_{1}^{T}$ is a white noise sequence with $v_{t} \sim N\left(0, \sigma_{v}^{2}\right)$. Furthermore, $y_{t}$ is given by

$$
y_{t}=x_{t}^{3}+u_{t}
$$

where $u_{t}$ is white noise with $u_{t} \sim N\left(0, \sigma_{u}^{2}\right)$. Figure 1 presents a realization of the sequences $x_{t}$ and $y_{t}$, where $\sigma_{v}^{2}=\sigma_{u}^{2}=0.01$. The sample size is $T=200$. Apparently, there is a fairly strong comovement between both series suggesting a stable long run relationship. However, applying an augmented Dickey-Fuller test (with four lagged differences and a constant) is applied to a linear cointegration regression of the form $y_{t}=\beta x_{t}+u_{t}^{*}$, the value of the $t$-statistic is -2.77 , which is insignificant with respect to the 0.05 significance level. In fact, the residual series of the linear cointegration regression (see Figure 2) does not look like a stationary series. On the other hand, if the cointegration regression is based on the underlying nonlinear relationship, that is $y_{t}$ is regressed on $x_{t}^{3}$, the resulting residual series resembles white noise (see Figure 3). Applying the rank tests suggested in Section 3 yield significant test statistics with respect to all reasonable 
significance levels. This example illustrates that ignoring the nonlinear nature of the cointegration relationship may lead to the misleading conclusion that there exists no long run relationship between the series.

The rest of the paper is organized as follows. In Section 2 we demonstrate that the parametric cointegration test based on the residuals of a linear cointegration regression is inconsistent against a given class of nonlinear alternatives. Two rank statistics for a test against the null hypothesis of no cointegration are suggested in Section 3 and in Section 4 we consider the power of tests. Generalizations of the rank test procedures are proposed in Section 5 and a rank test for neglected nonlinearities in the cointegration relationship is suggested in Section 6. Section 7 presents some results on the small sample properties of the test procedures and Section 8 provides an application of the rank tests to the term structure of interest yields. Finally, Section 9 concludes.

\section{The properties of linear cointegration tests}

For a theoretical analysis of a nonlinear cointegration relationship, different concepts are used. Granger and Hallman (1991a) and Granger (1995) consider time series which are long memory in mean but have a nonlinear relationship which is short memory in mean. Corradi (1995) consider non-strong mixing processes (processes with a long memory) and strong mixing processes (short memory). In this paper we adopt the definition of an integrated process due to Phillips (1987). The degree of integration is defined as follows:

Definition 1: (i) A time series $z_{t}$ is I(0) if, as $T \rightarrow \infty$,

$$
\frac{1}{\sqrt{T}} \sum_{t=1}^{[r T]} z_{t} \Rightarrow \bar{\sigma}_{z} W(r),
$$

where $\bar{\sigma}_{z}^{2}=\lim _{T \rightarrow \infty} E\left(T \bar{z}^{2}\right), \bar{z}=T^{-1} \sum_{t=1}^{T} z_{t}$, and $W(r)$ represents a standard Brownian motion. (ii) If $(1-B)^{d} z_{t} \sim I(0)$, then $z_{t}$ is integrated of order d, denoted as $z_{t} \sim I(d)$.

Different sets of sufficient conditions for $z_{t} \sim I(0)$ are given in Phillips (1987) 
and Phillips and Solo (1992), for example.

In this section we will consider the nonlinear relationship between two real valued time series $\left\{x_{t}\right\}_{1}^{T}$ and $\left\{y_{t}\right\}_{1}^{T}$

$$
y_{t}=f\left(x_{t}\right)+u_{t},
$$

where $y_{t} \sim I(1)$ and $z_{t}=f\left(x_{t}\right) \sim I(1)$. Under the null hypothesis $u_{t}$ is assumed to be $I(1)$, whereas under the alternative of a cointegration relationship, $u_{t} \sim I(0)$.

As demonstrated by Granger and Hallman (1991b), the Dickey-Fuller test may perform poorly when applied to a nonlinear transformation of a random walk. To investigate the effects of a nonlinear cointegration relationship on the power of a residual based cointegration test, it is convenient to consider a variant of the Dickey-Fuller type test due to Sargan and Bhargava (1983) and Phillips and Oularis ${ }^{1}$ (1990). The statistic is given by

$$
S_{T}^{2}=\frac{1}{\omega_{11 \cdot 2} T^{2}} \sum_{t=1}^{T}\left(y_{t}-\hat{\beta} x_{t}\right)^{2},
$$

where the $\hat{\beta}$ is the least-squares estimator from a regression of $y_{t}$ on $x_{t}$. The parameter $\omega_{11.2}$ is defined in Phillips and Oularis (1990, below eq. 12).

For the function $z=f(x)$ we make the following assumption:

Assumption 1: (i) The function $f(x)$ is monotonic increasing. (ii) There exist a function $h(a)$ such that $f^{-1}(a z)=h(a) f^{-1}(z)$, where $h^{-1}(z)$ indicates the inverse function.

Instead of (i) we may alternatively assume that the function is monotonic decreasing. Assumption (ii) is unnecessarily restrictive and can be replaced by an assumption that ensures that the asymptotic behavior of $f^{-1}\left(a_{T} z_{T}\right)$ is dominated by a term like $h\left(a_{T}\right) f^{-1}\left(z_{T}\right)$, as $T \rightarrow \infty$. However, to fascilitate the proof of the following theorem we assume that (ii) holds exactly for all values of $a$.

\footnotetext{
${ }^{1}$ The Sargan-Bhargava statistic equals the inverse of the variance ratio statistic suggested by Phillips and Oularis.
} 
Theorem 1: Let $z_{t}=f\left(x_{t}\right)=\sum_{i=1}^{t} v_{i}$ be integrated of order one and $y_{t}$ is generated as in (2), where $u_{t} \sim I(0)$. Under Assumption 1, a test based on the statistic $S_{T}^{2}$ given in (3) is consistent if and only if $f(x)$ is a linear function.

PROOF: Using

$$
\frac{1}{T^{2}} \sum_{t=1}^{T} \hat{u}_{t}^{2}=\frac{1}{T^{2}} \sum_{t=1}^{T} y_{t}^{2}-\frac{\left(T^{-2} \sum_{t=1}^{T} y_{t} x_{t}\right)^{2}}{T^{-2} \sum_{t=1}^{T} x_{t}^{2}}
$$

the test is seen to be consistent if the difference on the right hand side converge to zero as $T \rightarrow \infty$. From Assumption 1 and the continuous mapping theorem it follows that

$$
T^{-2} \sum_{t=1}^{T} y_{t}^{2}=T^{-2} \sum_{t=1}^{T}\left(z_{t}+u_{t}\right)^{2} \Rightarrow \bar{\sigma}_{z}^{2} \int W(r)^{2} \mathrm{~d} r .
$$

Furthermore,

$$
\begin{aligned}
T^{-2} \sum_{t=1}^{T} x_{t}^{2} & =T^{-2} \sum_{t=1}^{T} f^{-1}\left(z_{t}\right)^{2} \\
& =h\left(\bar{\sigma}_{z} T^{-1 / 2}\right)^{-2} T^{-2} \sum_{t=1}^{T} f^{-1}\left(\bar{\sigma}_{z} T^{-1 / 2} z_{t}\right)^{2} \\
& \Rightarrow h\left(\bar{\sigma}_{z} T^{-1 / 2}\right)^{-2} \int f^{-1}[W(r)]^{2} \mathrm{~d} r
\end{aligned}
$$

and

$$
\begin{aligned}
T^{-2} \sum_{t=1}^{T} y_{t} x_{t} & =T^{-2} \sum_{t=1}^{T} z_{t} f^{-1}\left(z_{t}\right)+o_{p}(1) \\
& =h\left(\bar{\sigma}_{z} T^{-1 / 2}\right)^{-1} T^{-2} \sum_{t=1}^{T} z_{t} f^{-1}\left(\sigma_{z} T^{-1 / 2} z_{t}\right) \\
& \Rightarrow \bar{\sigma}_{z} h\left(\bar{\sigma}_{z} T^{-1 / 2}\right)^{-1} \int W(r) f^{-1}[W(r)] . \mathrm{d} r
\end{aligned}
$$

It follows that

$$
\frac{1}{T^{2}} \sum_{t=1}^{T} \hat{u}_{t}^{2} \Rightarrow \bar{\sigma}_{z}^{2} \int W(r)^{2} \mathrm{~d} r-\bar{\sigma}_{z}^{2} \frac{\left\{\int W(r) f^{-1}[W(r)] \mathrm{d} r\right\}^{2}}{\int f^{-1}[W(r)]^{2} \mathrm{~d} r} .
$$

Since $x=f^{-1}(z)$ is an affine mapping it is seen that the right hand side of (5) is zero iff $f(x)=b x$ with some constant $b$. 
This Theorem shows that residual based cointegration tests are inconsistent for the class of functions given by Assumption 1. With some straightforward modifications the result extends to other unit root test like the original DickeyFuller test.

An example may help to illustrate the result. Let $y_{t}$ be generated as in the example of the introduction given in eq. (1). Using (5) and $f^{-1}(z)=x^{1 / 3}$ we have for $\beta \neq 0$ :

$$
S_{T}^{2} \Rightarrow \int W(r)^{2} \mathrm{~d} r-\frac{\left[\int W(r)^{4 / 3} \mathrm{~d} r\right]^{2}}{\int W(r)^{2 / 3} \mathrm{~d} r}
$$

Thus, under the alternative of nonlinear cointegration, the test statistic is $O_{p}(1)$. Accordingly, a test based on $S_{T}$ is inconsistent against nonlinear cointegration relationship as given in (1).

\section{A rank test for cointegration}

To overcome the difficulties of standard unit root test to detect nonlinear cointegration, we apply a rank transformation to the time series. The resultant test is valid for slightly more general situations as considered in the previous section. Specifically, we assume under the alternative that there exists a nonlinear cointegration relationship given by

$$
u_{t}=g\left(y_{t}\right)-f\left(x_{t}\right)
$$

where $f\left(x_{t}\right) \sim I(1), g\left(y_{t}\right) \sim I(1)$ and $u_{t} \sim I(0)$. The functions $g(y)$ and $f(x)$ are monotonic increasing. If it is not known whether these functions are monotonic increasing or decreasing, a two-sided test is available. A similar framework is considered in Granger and Hallman (1991a).

We define the ranked series as

$$
R_{T}\left(x_{t}\right)=\operatorname{Rank}\left[\text { of } x_{t} \text { among } x_{1}, \ldots, x_{T}\right]
$$

and construct $R_{T}\left(y_{t}\right)$ accordingly. In Breitung and Gouriéroux (1997), an asymptotic theory for a ranked random walk is developed. Here we give a slightly more general version of their main result. 
Theorem 2: Let $x_{t}=\mu+\sum_{i=1}^{T} v_{i}$ be I(1) as defined in Definition 1. Then, as $T \rightarrow \infty$, the limiting distribution of the sequence of ranks can be represented as

$$
T^{-1} R_{T}\left(x_{[a T]}\right) \Rightarrow a \mathcal{A}_{1}+(1-a) \mathcal{A}_{2}
$$

where $\mathcal{A}_{1}$ and $\mathcal{A}_{2}$ are two independent random variables with an arcsine distribution.

Proof: Consider

$$
\begin{aligned}
T^{-1} R_{T}\left(x_{[a T]}\right) & =T^{-1} \sum_{t=1}^{T} \mathbb{I}\left(x_{t}<x_{[a T]}\right) \\
& =\sum_{t} \mathbb{I}\left(\frac{1}{\sqrt{T}} z_{\left[\frac{t}{T} T\right]}<\frac{1}{\sqrt{T}} z_{[a T]}\right)\left[\frac{t}{T}-\frac{t-1}{T}\right] \\
& \Rightarrow \int_{0}^{1} \mathbb{I}[W(u)<W(a)] \mathrm{d} u \\
& =\int_{0}^{a} \mathbb{I}[W(u)<W(a)] \mathrm{d} u+\int_{a}^{1} \mathbb{I}[W(u)<W(a)] \mathrm{d} u .
\end{aligned}
$$

Since the increments of the Brownian motion are independent, the two parts of the integral are independent as well.

Using $\stackrel{d}{=}$ to indicate equality in distribution we have

$$
\begin{aligned}
\int_{0}^{a} \mathbb{I}[W(u)<W(a)] \mathrm{d} u & =\int_{0}^{a} \mathbb{I}[W(a)-W(u)>0] \mathrm{d} u \\
& \stackrel{d}{=} \int_{0}^{a} \mathbb{I}[W(a-u)>0] \mathrm{d} u \\
& \stackrel{d}{=} a \int_{0}^{1} \mathbb{I}[W(u)>0] \mathrm{d} u \\
& \stackrel{d}{=} a \mathcal{A}_{1},
\end{aligned}
$$

where $\mathcal{A}_{1}=\int_{0}^{1} \mathbb{I}[W(u)>0] \mathrm{d} u$ is a random variable with an arcsine distribution (cf. Breitung and Gouriéroux, 1997). Similarly, we find

$$
\int_{a}^{1} \mathbb{I}[W(u)<W(a)] \mathrm{d} u=(1-a) \mathcal{A}_{2}
$$

where $\mathcal{A}_{2}$ is another random variable with an arcsine distribution independent of $\mathcal{A}_{1}$.

The rank statistic is constructed by replacing $f\left(x_{t}\right)$ and $g\left(y_{t}\right)$ by $R_{T}\left(x_{t}\right)$ and $R_{T}\left(y_{t}\right)$, respectively. Since we assume that $f\left(x_{t}\right)$ and $g\left(y_{t}\right)$ are two random walk 
series, it follows that $R_{T}\left(x_{t}\right)=R_{T}\left[f\left(x_{t}\right)\right]$ and $R_{T}\left(y_{t}\right)=R_{T}\left[g\left(y_{t}\right)\right]$ behave like ranked random walks for which the limiting distribution is given in Theorem 2. The advantage of a statistic based on the sequence of ranks is that the functions $f(\cdot)$ and $g(\cdot)$ need not be known.

We consider two "distance measures" between the sequences $R_{T}\left(x_{t}\right)$ and $R_{T}\left(y_{t}\right):$

$$
\begin{aligned}
\kappa_{T} & =T^{-1} \sup _{t}\left|d_{t}\right| \\
\xi_{T} & =T^{-3} \sum_{t=1}^{T} d_{t}^{2},
\end{aligned}
$$

where $d_{t}=R_{T}\left(y_{t}\right)-R_{T}\left(x_{t}\right)$. It should be noted that $d_{t}$ is $O_{p}(T)$ and, thus, the normalization factors are different from other applications of these measures. The statistic $\kappa_{T}$ is a Kolmogorov-Smirnov type of statistic considered by Lo (1991) and $\xi_{T}$ is a Cramer-von-Mises type of statistic used by Sargan and Bhargava (1983). The null hypothesis of no (nonlinear) cointegration between $x_{t}$ and $y_{t}$ is rejected if the test statistics are too small.

It is interesting to note that the statistic $\xi_{T}$ allows for different interpretations. Let $\tilde{b}_{T}$ denote the least-squares estimate from a regression of $R_{T}\left(y_{t}\right)$ on $R_{T}\left(x_{t}\right)$. Using $\sum R_{T}\left(x_{t}\right)^{2}=\sum R_{T}\left(y_{t}\right)^{2}=T^{3} / 3+O\left(T^{2}\right)$ we have

$$
\begin{aligned}
\xi_{T} & =\frac{1}{T^{3}} \sum_{t=1}^{T}\left[R_{T}\left(y_{t}\right)^{2}-2 R_{T}\left(y_{t}\right) R_{T}\left(x_{t}\right)+R_{T}\left(x_{t}\right)^{2}\right] \\
& =\frac{2-2 \tilde{b}_{T}}{T^{3}} \sum_{t=1}^{T} R_{T}\left(x_{t}\right)^{2} \\
& =\frac{2}{3}\left(1-\tilde{b}_{T}\right)+o_{p}(1) .
\end{aligned}
$$

If $y_{t}$ and $x_{t}$ are not cointegrated, then $\tilde{b}_{T}$ has a nondegenerate limiting distribution (see Phillips (1986) for the linear case). On the other hand, if $y_{t}$ and $x_{t}$ are cointegrated, then $\tilde{b}_{T}$ converges to one in probability and therefore $\xi_{T}$ converges to zero.

Second, consider a Cramer-von Mises type of statistic based on the residuals of a cointegration regression on the ranks:

$$
\widetilde{\xi}_{T}=\frac{1}{T^{3}} \sum_{t=1}^{T}\left[R_{T}\left(y_{t}\right)-\tilde{b}_{T} R_{T}\left(x_{t}\right)\right]^{2}
$$




$$
\begin{aligned}
& =\frac{1}{T^{3}} \sum_{t=1}^{T}\left[R_{T}\left(y_{t}\right)^{2}-2 \tilde{b}_{T} R_{T}\left(y_{t}\right) R_{T}\left(x_{t}\right)+\tilde{b}_{T}^{2} R_{T}\left(x_{t}\right)^{2}\right] \\
& =\frac{1-\tilde{b}_{T}^{2}}{T^{3}} \sum_{t=1}^{T} R_{T}\left(x_{t}\right)^{2} \\
& =\frac{1}{3}\left(1-\tilde{b}_{T}^{2}\right)+o_{p}(1) .
\end{aligned}
$$

Hence, a two-step approach similar to the one suggested by Engle and Granger (1987) can be seen as a two-sided version of a test based on $\xi_{T}$.

Third, the statistic $\xi_{T}$ is related to the rank correlation coefficient, which is known as "Spearman's rho". Spearman's rho is defined as

$$
r_{s}=1-\frac{6}{T^{3}-T} \sum_{t=1}^{T} d_{t}^{2}
$$

(eg Kendall and Gibbons (1990, p.8)). The statistic $r_{s}$ can therefore be seen as a mapping of $\xi_{T}$ into the interval $[-1,1]$. If $x_{t}$ and $y_{t}$ are cointegrated, Spearman's rho converges in probability to one as $T \rightarrow \infty$.

Theorem 2 implies that, if $f\left(x_{t}\right)$ and $g\left(y_{t}\right)$ are independent random walk sequences, we have

$$
T^{-1} d_{[a T]} \Rightarrow a\left(\mathcal{A}_{1}-\mathcal{A}_{3}\right)+(1-a)\left(\mathcal{A}_{2}-\mathcal{A}_{4}\right)
$$

where $\mathcal{A}_{1}, \ldots, \mathcal{A}_{4}$ are independent random variables with an arcsine distribution. Hence, the increments of random walk sequences $x_{t}$ and $y_{t}$ may be allowed to be heteroscedastic and serially correlated.

\section{Power}

Under the alternative of a cointegration relationship as given in (6) we have

$$
\begin{aligned}
T^{-1} d_{[a T]} & =T^{-1}\left\{R_{T}\left[T^{-1 / 2} g\left(y_{t}\right)\right]-R_{T}\left[T^{-1 / 2} f\left(x_{t}\right)\right]\right\} \\
& =T^{-1}\left\{R_{T}\left[T^{-1 / 2} f\left(x_{t}\right)+o_{p}(1)\right]-R_{T}\left[T^{-1 / 2} f\left(x_{t}\right)\right]\right\} \\
& \Rightarrow 0 .
\end{aligned}
$$

Hence, $\kappa_{T}$ and $\xi_{T}$ converge to zero as $T \rightarrow \infty$, i.e., both rank tests are consistent. 
Apart from this general statement it is quite difficult to obtain analytical results for the (local) power of the test. Nevertheless, some interesting properties of the rank test can be observed when the parametric analog of the ranked differences is considered. Let the normalized difference of the series be defined as

$$
\delta_{t}=\frac{y_{t}}{\bar{\sigma}_{y}}-\frac{x_{t}}{\bar{\sigma}_{x}}
$$

where $y_{t}$ and $x_{t}$ are $I(1), E\left(y_{t}\right)=E\left(x_{t}\right)=0$ for all $t$, and $\bar{\sigma}_{x}^{2}, \bar{\sigma}_{y}^{2}$ denote the respective long-run variances (cf Definition 1). Accordingly, a parametric analog of the statistic $\xi_{T}$ is constructed as

$$
D_{T}=\frac{1}{T^{2}} \sum_{t=1}^{T} \delta_{t}^{2}
$$

and under the null hypothesis of two uncorrelated random walk sequences, the statistic is asymptotically distributed as $4 \int W(r)^{2} \mathrm{~d} r$. Under the alternative hypothesis assume that $u_{t}=y_{t}-\beta x_{t}$ is stationary and $\beta>0$. In this case we have $\bar{\sigma}_{y}=\beta \bar{\sigma}_{x}$ so that

$$
D_{T}=\frac{1}{T^{2}} \sum_{t=1}^{T}\left(\frac{\beta x_{t}+u_{t}}{\beta \bar{\sigma}_{x}}-\frac{x_{t}}{\bar{\sigma}_{x}}\right)^{2}=\frac{1}{\beta^{2} \bar{\sigma}_{x}^{2} T^{2}} \sum_{t=1}^{T} u^{2} .
$$

It is seen that for large $T$ the power of the test depends on the "signal-to-noise ratio" $\beta^{2} \bar{\sigma}_{x}^{2} / \sigma_{u}^{2}$, where $\sigma_{u}^{2}$ is the variance of $u_{t}$. As a consequence, the power of the test is a monotonic increasing function of the parameter $\beta$. In contrast, $\beta$ does not affect the power of a Dickey-Fuller cointegration test. As a result, we expect that a test based on $D_{T}$ or its ranked counterpart $\xi_{T}$ will have more (less) power than the Dickey-Fuller type cointegration test if $\beta$ is large (small).

\section{Extensions}

The asymptotic theory for the case that $x_{t}$ and $y_{t}$ are mutually correlated random walk sequences turns out to be much more complicated. The problem is that in this case the limiting distributions generally depend on the functions $f\left(x_{t}\right)$ and $g\left(y_{t}\right)$ which is assumed to be unknown. In practice, however, we may hope that it 
is sufficient to correct for the linear effects of the correlation and that the impact of nonlinearity to the limiting distribution is small.

Assuming that $x_{t}$ and $y_{t}$ are linear random walks with zero means and serially uncorrelated increments, the long run variances are identical to the usual variances of the increments, that is,

$$
\begin{gathered}
\bar{\sigma}_{x}^{2}=\sigma_{\Delta x}^{2}=T^{-1} \sum_{t=1}^{T} E\left(\Delta x_{t}\right)^{2} \\
\bar{\sigma}_{y}^{2}=\sigma_{\Delta y}^{2}=T^{-1} \sum_{t=1}^{T} E\left(\Delta y_{t}\right)^{2},
\end{gathered}
$$

where $\Delta y_{t}$ denotes the first difference operator such that $\Delta x_{t}=x_{t}-x_{t-1}$ and it is assumed that $x_{0}=y_{0}=0$. Furthermore, the covariance between $\Delta x_{t}$ and $\Delta y_{t}$ is

$$
\sigma_{\Delta x \Delta y}=T^{-1} \sum_{t=1}^{T} E\left(\Delta x_{t} \Delta y_{t}\right)
$$

so that $\sigma_{\Delta \delta}^{2}=\sigma_{\Delta y}^{2}-2 \sigma_{\Delta x \Delta y}+\sigma_{\Delta x}^{2}$. Under the null hypothesis of mutually correlated random walks, the corrected test statistic

$$
D_{T}^{*}=\frac{1}{\hat{\sigma}_{\Delta \delta}^{2} T^{2}} \sum_{t=1}^{T} \delta_{t}^{2}
$$

with

$$
\hat{\sigma}_{\Delta \delta}^{2}=\frac{1}{T} \sum_{t=2}^{T}\left(\Delta \delta_{t}\right)^{2}
$$

is asymptotically distributed as $\int W(r)^{2} \mathrm{~d} r$ and, thus, does not depend on nuisance parameters.

Ignoring the nonlinear nature of the rank transformation we can similarly construct corrected rank statistics as

$$
\begin{aligned}
\xi_{T}^{*} & =\xi_{T} / \hat{\sigma}_{\Delta d}^{2} \\
\kappa_{T}^{*} & =\kappa_{T} / \hat{\sigma}_{\Delta d},
\end{aligned}
$$

where

$$
\hat{\sigma}_{\Delta d}^{2}=T^{-1} \sum_{t=2}^{T}\left(\Delta d_{t}\right)^{2}
$$


Although there is no proper asymptotic justification for this correction in case of nonlinear transformations, Monte Carlo simulations suggest that the corrections performs reasonably well in practice.

Similar properties are obtained by using the Dickey-Fuller statistic to test the residuals of a ranked cointegration regression. It is well known that in the linear case the limiting null distribution of the Dickey-Fuller test for the cointegration residuals does not depend on the correlation between the increments of the random walk series (cf Phillips and Oularis (1990)). One may therefore hope that such a test may be useful for the nonlinear case as well.

Furthermore this test can be straightforwardly generalized to cointegration among the $k+1$ variables $y_{t}, x_{1 t}, \ldots, x_{k t}$, where it is assumed that $g\left(y_{t}\right)$ and $f_{j}\left(x_{j t}\right)$ $(j=1, \ldots, k)$ are monotonic functions. Let $R_{T}\left(\mathbf{x}_{t}\right)=\left[R_{T}\left(x_{1 t}\right), \ldots, R_{T}\left(x_{k t}\right)\right]^{\prime}$ be a $k \times 1$ vector and $\tilde{\mathbf{b}}_{T}$ is the least-squares estimate from a regression of $R_{T}\left(y_{t}\right)$ on $R_{T}\left(\mathbf{x}_{t}\right)$. A ranked Dickey-Fuller test for cointegration is obtained by testing the hypothesis $\rho=0$ in the regression

$$
\Delta \tilde{u}_{t}=\rho \tilde{u}_{t-1}+e_{t}
$$

where

$$
\tilde{u}_{t}=R_{T}\left(y_{t}\right)-\tilde{\mathbf{b}}_{T}^{\prime} R_{T}\left(\mathbf{x}_{t}\right)
$$

The corresponding $t$-statistic is denoted by $\widetilde{Q}_{T}[k]$. Using Monte Carlo simulations, the critical values for the test statistics $\kappa_{T}, \xi_{T}, \kappa_{T}^{*}, \xi_{T}^{*}$, and $\widetilde{Q}_{T}[k]$ are computed for $T=100$ and $T=500$ (see Table 1 ).

\section{$6 \quad$ A rank test for neglected nonlinearity}

Whenever the rank test for cointegration indicates a stable long run relationship, it is interesting to know whether the cointegration relationship is linear or nonlinear. For a convenient representation of such null and alternative hypotheses we follow Granger (1995) and write the nonlinear relationship as

$$
y_{t}=\gamma_{0}+\gamma_{1} x_{t}+f^{*}\left(x_{t}\right)+u_{t}
$$


where $\gamma_{0}+\gamma_{1} x_{t}$ is the linear part of the relationship. Under the null hypothesis it is assumed that $f^{*}\left(x_{t}\right)=0$ for all $t$. If $f^{*}\left(x_{t}\right)$ is unknown, it may be approximated by Fourier series (Gallant (1981)) or a neuronal network (Lee, White and Granger (1993)). Here we suggest to use the multiple of the rank transformation $\theta R_{T}\left(x_{t}\right)$ instead of $f^{*}\left(x_{t}\right)$.

It is interesting to note that the rank transformation is to some extent related to the neural network approach suggested by Lee, White and Granger (1993). If $\mathbf{x}_{\mathbf{t}}$ is a $k \times 1$ vector of "input variables" and $\alpha$ is a corresponding vector of coefficients, the neural network approach approximates $f^{*}\left(\mathbf{x}_{\mathbf{t}}\right)$ by $\sum_{j=1}^{q} \beta_{j} \psi\left(\mathbf{x}_{t}^{\prime} \alpha_{j}\right)$, where $\psi(\cdot)$ has the properties of a cumulated distribution function. A function often used in practice is the logistic $\psi(x)=x /(1-x)$. In our context, $x_{t}$ is a scalar variable, so that the neuronal network term simplifies to $\beta \psi\left(\alpha x_{t}\right)$. Using $T^{-1} R_{T}\left(x_{t}\right)=\widehat{F}_{T}\left(x_{t}\right)$, where $\widehat{F}_{T}\left(x_{t}\right)$ is the empirical distribution function, the rank transformation can be motivated as letting $\psi\left(\alpha x_{t}\right)$ be the empirical distribution function with the attractive property that the parameter $\alpha$ can be dropped due to the invariance of the rank transformation.

If it is assumed that $x_{t}$ is exogenous and $u_{t}$ is white noise with $u_{t} \sim N\left(0, \sigma^{2}\right)$, a score test statistic is obtained as the $T \cdot R^{2}$-statistic of the least-squares regression

$$
\tilde{u}_{t}=c_{0}+c_{1} x_{t}+c_{2} R_{T}\left(x_{t}\right)+e_{t}
$$

where $\tilde{u}_{t}=y_{t}-\tilde{\gamma}_{0}-\tilde{\gamma}_{1} x_{t}$ and $\tilde{\gamma}_{0}$ and $\tilde{\gamma}_{1}$ are the least-squares estimates from a regression of $y_{t}$ on a constant and $x_{t}$.

A problem with applying the usual asymptotic theory to derive the limiting null distribution of the test statistic is that the regression (15) involves the nonstationary variables $x_{t}$ and $R_{T}\left(x_{t}\right)$. However under some (fairly restrictive) assumptions, Theorem 3 shows that under the null hypothesis $c_{2}=0$ the score statistic is asymptotically $\chi^{2}$ distributed.

Theorem 3: Let $x_{t}=\sum_{j=1}^{t} v_{j}$ and

$$
y_{t}=\gamma_{0}+\gamma_{1} x_{t}+u_{t}
$$

where it is assumed that $v_{t}$ is $I(0)$ according to Definition 1 and $u_{t}$ is white noise 
with $E\left(u_{t}\right)=0$ and $E\left(u_{t}^{2}\right)=\sigma_{u}^{2}$. As $T \rightarrow \infty$, the score statistic for $H_{0}: c_{2}=0$ in the regression (15) has an asymptotic $\chi^{2}$ distribution with one degree of freedom.

Proof: It is convenient to introduce the matrix notation:

$$
\mathbf{X}_{\mathbf{1}}=\left[\begin{array}{cc}
1 & x_{1} \\
\vdots & \vdots \\
1 & x_{T}
\end{array}\right] \quad \text { and } \mathbf{X}_{\mathbf{2}}=\left[\begin{array}{c}
R_{T}\left(x_{1}\right) \\
\vdots \\
R_{T}\left(x_{T}\right)
\end{array}\right]
$$

$\mathbf{y}=\left[y_{1}, \ldots, y_{T}\right]^{\prime}$ and $\tilde{\mathbf{u}}=\left[\tilde{u}_{1}, \ldots, \tilde{u}_{T}\right]^{\prime}$. With this notation, the score statistic can be written as

$$
T \cdot R^{2}=\frac{1}{\tilde{\sigma}^{2}}\left(\hat{\beta}_{2}\right)^{2}\left[\mathbf{X}_{\mathbf{2}}^{\prime} \mathbf{X}_{\mathbf{2}}-\mathbf{X}_{\mathbf{2}}^{\prime} \mathbf{X}_{\mathbf{1}}\left(\mathbf{X}_{\mathbf{1}}^{\prime} \mathbf{X}_{\mathbf{1}}\right)^{-\mathbf{1}} \mathbf{X}_{\mathbf{1}}^{\prime} \mathbf{X}_{\mathbf{2}}\right]
$$

where $\hat{\beta}_{2}$ is the least-squares estimator of $\beta_{2}$ in the regression $\mathbf{y}=\mathbf{X}_{\mathbf{1}} \beta_{1}+\mathbf{X}_{\mathbf{2}} \beta_{2}+$ $\mathbf{u}$ and $\tilde{\sigma}^{2}=\tilde{\mathbf{u}}^{\prime} \tilde{\mathbf{u}} / T$. As shown by Park and Phillips (1988), the least-square estimator in a regression with strictly exogenous $I(1)$ regressors is conditionally normally distributed, so that conditional on $\mathbf{X}=\left[\mathbf{X}_{\mathbf{1}}, \mathbf{X}_{\mathbf{2}}\right], \tilde{\beta}_{2}$ is asymptotically distributed as $N\left(\mathbf{0}, \mathbf{V}_{\mathbf{2}}\right)$, where

$$
\mathbf{V}_{\mathbf{2}}=\sigma_{u}^{2}\left[\mathbf{X}_{\mathbf{2}}^{\prime} \mathbf{X}_{\mathbf{2}}-\mathbf{X}_{\mathbf{2}}^{\prime} \mathbf{X}_{\mathbf{1}}\left(\mathbf{X}_{\mathbf{1}}^{\prime} \mathbf{X}_{\mathbf{1}}\right)^{-\mathbf{1}} \mathbf{X}_{\mathbf{1}}^{\prime} \mathbf{X}_{\mathbf{2}}\right]^{-1}
$$

From $\tilde{\sigma}_{u}^{2} \stackrel{p}{\longrightarrow} \sigma_{u}^{2}$ it follows that $T \cdot R^{2}$ has an asymptotic $\chi^{2}$ distribution with one degree of freedom.

Unfortunately, the assumptions for Theorem 3 are too restrictive to provide a useful result for practical situations. In many situations, the errors $u_{t}$ are found to be serially correlated and the regressor $x_{t}$ may be endogenous. However, using standard techniques for cointegration regressions (Saikkonen (1991), Stock and Watson (1993)) the test can be modified to accommodate serially correlated errors and endogenous regressors. For this purpose assume that

$$
\begin{aligned}
u_{t} & =E\left(u_{t} \mid \Delta x_{t}, \Delta x_{t \pm 1}, \Delta x_{t \pm 2}, \ldots\right)+v_{t} \\
& =\sum_{j=-\infty}^{\infty} \pi_{j} \Delta x_{t-j}+v_{t}
\end{aligned}
$$


and $v_{t}$ admits the autoregressive representation

$$
v_{t}=\sum_{j=1}^{\infty} \alpha_{j} v_{t-j}+\varepsilon_{t},
$$

where the lag polynomial $\alpha(B)=1-\alpha_{1} B-\alpha_{2} B^{2}-\cdots$ has all roots outside the complex unit circle.

Under the null hypothesis of linear cointegration we thus have the representation

$$
y_{t}=\gamma_{0}^{*}+\sum_{j=1}^{\infty} \alpha_{j} y_{t-j}+\gamma_{1}^{*} x_{t}+\sum_{j=-\infty}^{\infty} \pi_{j}^{*} \Delta x_{t-j}+\varepsilon_{t},
$$

(see Stock and Watson (1993) and Inder (1995)).

A test for non-linear cointegration can be obtained by truncating the infinite sums appropriately and forming $T \cdot R^{2}$ for the regression of the residuals $\tilde{\varepsilon}_{t}$ on the regressors of (16) and $R_{T}\left(x_{t}\right)$. Along the lines of Theorem 3 it can be shown that the resulting score statistic is asymptotically $\chi^{2}$ distributed under the null hypothesis of a linear cointegration relationship.

\section{$7 \quad$ Small sample properties}

To investigate the small sample properties of the rank tests we follow Gonzalo (1994) and generate two time series according to the model equations

$$
\begin{aligned}
& y_{t}=\beta z_{t}+u_{t}, \quad u_{t}=\alpha u_{t-1}+\varepsilon_{t} \\
& z_{t}=z_{t-1}+v_{t}
\end{aligned}
$$

where

$$
\left[\begin{array}{l}
\varepsilon_{t} \\
v_{t}
\end{array}\right] \sim \operatorname{iid} N\left(\mathbf{0},\left[\begin{array}{ll}
1 & \rho \\
\rho & 1
\end{array}\right]\right) .
$$

The variable $x_{t}$ is obtained from the random walk $z_{t}$ by using the inverse function $x_{t}=f^{-1}\left(z_{t}\right)$.

Under the null hypothesis $H_{0}: \alpha=1$ there is no cointegration relationship between the series. If in addition $\beta=0$ and $\rho=0$, then $x_{t}$ and $y_{t}$ are two independent random walks with serially uncorrelated increments. For this specification, Table 2 reports the rejection frequencies of different cointegration tests. 
The rank tests $\kappa_{T}$ and $\xi_{T}$ are computed as in (7) and (8) and "CDF" indicates the Dickey-Fuller $t$-test applied to the residuals of a linear regression of $y_{t}$ on $x_{t}$ and a constant. The results for the linear process is given in the left half of Table 2 indicated by $f(x)=x$. It might be surprising to see that for $\beta=1$ the rank test is much more powerful than the CDF test if $\alpha$ is close to one. As was argued in Section 4, this is due to the fact that the rank statistics depend on the parameter $\beta$, whereas the Dickey-Fuller type test for cointegration does not depend on $\beta$. In fact, the simulation results indicate that the power of the rank tests are quite sensitive to the value of $\beta$. For $\beta=0.5$ the Dickey-Fuller tests perform better, whereas for $\beta=1$ the rank tests clearly outperform the Dickey-Fuller type of tests.

It should also be noted that the rank test does not require to estimate the cointegration parameter $\beta$. Accordingly, this test has the same power as for the case of a known cointegration relationship. Furthermore, the rank test procedures impose the one-sided hypothesis that $f\left(x_{t}\right)$ is an increasing function.

Since the rank tests are invariant to a monotonic transformation of the variables, the power function is the same as for the linear case. Comparing the power of the CDF test with the rank counterparts, it turns out that the power of the CDF test may drop dramatically for nonlinear alternatives (see also Granger and Hallman (1991b)), while the rank test performs as well as in the linear case. In particular, for the case $f(x)=\log (x)$ the parametric CDF test performs quite poorly.

To investigate the power of the rank test against nonlinear alternatives, which cannot be written as in (6) we generate data using the threshold cointegrated process given by

$$
u_{t}= \begin{cases}u_{t-1}+\varepsilon_{t} & \text { if }\left|u_{t-1}\right| \leq \lambda \sigma_{u} \\ a u_{t-1}+\varepsilon_{t} & \text { if }\left|u_{t-1}\right|>\lambda \sigma_{u}\end{cases}
$$

where $\sigma_{u}$ is the standard deviation of $u_{t}$ and $\lambda$ is a positive constant. Furthermore we generate $z_{t}$ as a random walk independent of $u_{t}$ and let $\beta=1$. Such nonlinear processes are investigated by Balke and Fomby (1997). We consider two different threshold values given by $\lambda=0.5$ and $\lambda=1.5$. The results for various values of 
$a$ are presented in Table 3. It turns out that the rank tests possess much more power against such kind of nonlinear alternatives than the CDF test.

As noted in Section 5, the rank tests cannot be applied to test the null hypothesis of two correlated random walks. Since the corrections suggested in Section 5 lacks a precise asymptotic justification, it is interesting to consider the performance of the corrected test statistics for different values of $\rho$. Table 4 presents the empirical sizes for testing the null hypothesis of no cointegration. It turns out that for a substantial correlation all tests possess a serious size bias. Among the rank tests, the statistic $\kappa_{T}^{*}$ turns out to perform best. In sum, the results suggest that the correction works satisfactory for small and moderate correlation only.

Next we consider the small sample properties of the rank test for nonlinear cointegration suggested in Section 6. It is assumed that $y_{t}$ and $x_{t}$ are cointegrated so that $y_{t}-\beta f\left(x_{t}\right)$ is stationary. By setting $\alpha=0.5$ we generate serially correlated errors and letting $\rho=0.5$, the variable $x_{t}$ is correlated with the errors $u_{t}$, that is, $x_{t}$ is endogenous. The rank test for nonlinear cointegration is obtained by regressing $y_{t}$ on $x_{t}, y_{t-1}, \Delta x_{t+1}, \Delta x_{t}, \Delta x_{t-1}$ and a constant. The score statistic is computed as $T \cdot R^{2}$ from a regression of the residuals on the same set of regressors and the ranks $R_{T}\left(x_{t}\right)$.

To study the power of the test we consider three different nonlinear functions. As a benchmark we perform the tests using $f\left(x_{t}\right)$ instead of the ranks $R_{T}\left(x_{t}\right)$. Of course, using the true functional form, which is usually unknown in practice, we expect the test to have better power than the test based on the ranks. Surprisingly, the results of the Monte Carlo simulations (see Table 5) suggest that the rank test may even be (slightly) more powerful than the parametric test, whenever the nonlinear term enter the equation with a small weight $(\beta=0.01)$. However, the gain in power is quite small and fall in the range of the simulation error. In any case, the rank test performs very well and seems to imply no important loss of power in comparison to the parametric version of the test. 


\section{Empirical application}

The rank tests are applied to test for a possible nonlinear cointegration between interest rates with different time to maturity. Recent empirical work suggests that interest rates with a different time to maturity are nonlinearly related. ${ }^{2}$ The data set consists of monthly yields of government bonds with different time to maturity as published by the German Bundesbank. The sample runs from 1967(i) through 1995(xii) yielding 348 monthly observations for each variable.

The nonlinear relationship between yields for different times to maturity can be motivated as follows. Let $r_{t}$ denote the yield of a one-period bond and $R_{t}$ represents the yield of a two-period bond at time $t$. The expectation theory of the term structure implies that

$$
R_{t}=\phi_{t}+0.5 r_{t}+0.5 E_{t}\left(r_{t+1}\right)
$$

where $E_{t}$ denotes the conditional expectation with respect to the relevant information set available at period $t$ and $\phi_{t}$ represents the risk premium. Letting $r_{t+1}=E_{t}\left(r_{t+1}\right)+2 \nu_{t}$ and subtracting $r_{t}$ from both sides of (19) gives

$$
R_{t}-r_{t}=0.5\left(r_{t+1}-r_{t}\right)+\phi_{t}+\nu_{t}
$$

Assuming that $r_{t}$ is $I(1)$ and $\phi_{t}+\nu_{t}$ is stationary implies that $R_{t}$ and $r_{t}$ are (linearly) cointegrated (e.g. Wolters (1995)). However, if the risk premium depends on $r_{t}$ such that $\phi_{t}=f^{*}\left(r_{t}\right)+\eta_{t}$ with $\eta_{t}$ stationary, then the yields are nonlinearly cointegrated:

$$
R_{t}-f\left(r_{t}\right)=u_{t} \sim I(0),
$$

where $f\left(r_{t}\right)=r_{t}+f^{*}\left(r_{t}\right)$ and $u_{t}=0.5\left(r_{t+1}-r_{t}\right)+\eta_{t}+\nu_{t}$. Note that $u_{t}$ is correlated with $r_{t}$ and, therefore, $r_{t}$ is endogenous. Furthermore, if the sampling interval is shorter than the time to maturity, then the errors are serially correlated even if $\nu_{t}$ and $\eta_{t}$ are white noise.

To test whether interest rates possess a (nonlinear) cointegration relationship we first apply various tests for unit roots to the series. Neither the conventional Dickey-Fuller $t$-test nor the ranked counterpart suggested by Breitung and

\footnotetext{
${ }^{2}$ See, e.g., Campbell and Galbraight (1993), Pfann et al. (1996), and the reference therein.
} 
Gouriéroux (1997) reject the null hypothesis that the interest rates are $I(1)$ (not presented).

The results presented in Table 6 highlight the cointegration properties of yields with different time to maturity. The parametric as well as the rank tests indicate a cointegration relationship between the short term bonds, while the evidence for cointegration between short run and long run bonds (e.g. R1 and R10) is much weaker. Furthermore, the rank test for nonlinear cointegration ("nonlin") does not reveal evidence against a linear cointegration relationship. All values of the test statistic suggested in Section 6 are much smaller than the critical value of 3.84 .

\section{Concluding Remarks}

We have considered rank tests for a nonlinear cointegration relationship. Under the hypothesis that $T^{-1 / 2} g\left(y_{[a T]}\right)$ and $T^{-1 / 2} f\left(x_{[a T]}\right)$ converge weakly to two independent Brownian motions $W_{1}(a)$ and $W_{2}(a)$, the limiting distribution of the rank differences $d_{[a T]}=R_{T}\left(y_{[a T]}\right)-R_{T}\left(x_{[a T]}\right)$ can be derived. Tests based on the sequence $d_{t}$ appear to have good power properties against cointegration relationships of the form $g\left(y_{t}\right)-f\left(x_{t}\right)$. However, if the cointegration relationship has a more general form, for example $h\left(y_{t}, x_{t}\right)=x_{t} / y_{t} \sim I(0)$, the rank test may lack power. Therefore, it seems desirable to construct nonparametric tests against a more general form of nonlinear cointegration. Furthermore, we have no asymptotic theory for the case that $x_{t}$ and $y_{t}$ are correlated.

The results of our simulation experiments suggest that the rank tests may clearly outperform their parametric counterparts if the cointegration relationship is indeed nonlinear. However, if there is a substantial mutual correlation between the increments of the random walks, the rank tests may suffer from a size bias.

The rank test procedures were applied to test for nonlinear cointegration between interest yields in Germany. In all, our results do not reveal important nonlinearities for the relationships of of bonds with different time to maturity. 


\section{References}

Balke, N.S. and T.B. Fomby (1997), Threshold Cointegration, International Economic Review, 38, 627-645.

Breitung, J. and C. Gouriéroux (1997), Rank tests for unit roots, Journal of Econometrics, 81, 7-28.

Campbell, B. and J.W. Galbraith (1993), Inference in expectations models of the term structure: A non-parametric approach, Empirical Economics $18,623-638$.

Gallant, A.R. (1981), Unbiased Determination of Production Technologies, Journal of Econometrics, 30, 149-169.

Granger, C.W.J. (1981), Some properties of time series data and their use in econometric model specification, Journal of Econometrics, 16, 121-130.

Granger, C.W.J. (1986), Developments in the study of co-integrated economic variables, Oxford Bulletin of Economics and Statistics, 48, 213-228.

Granger, C.W.J. (1995), Modelling nonlinear relationships between extendedmemory variables, Econometrica, 63, 265-279.

Granger, C.W.J. and J. Hallman (1991a), Long memory processes with attractors, Oxford Bulletin of Economics and Statistics, 53, 11-26.

Granger, C.W.J. and J. Hallman (1991b), Nonlinear transformations of integrated time series, Journal of Time Series Analysis, 12, 207-224.

Granger, C.W.J., T. Inoue and N. Morin (1997), Nonlinear Stochastic Trends, Journal of Econometrics, 81, 65-92.

Granger, C.W.J. and T. Teräsvirta (1993), Modelling nonlinear economic relationships, Oxford: Oxford University Press.

Inder, B. (1995), Finite sample arguments for appropriate estimation of cointegrating vectors, discussion paper, Monash University, Australia. 
Kendall, M. and J.D. Gibbons (1990), Rank correlation methods, London: Edward Arnold.

Lee T.-H., White H., Granger C.W.J. (1993), Testing for neglected nonlinearity in time series models: a comparison of neural network methods and alternative tests, Journal of Econometrics, 56, 269-290.

Lo, A.W. (1991), Long-term memory in stock market prices, Econometrica, 59, 1279-1314.

Park, J.Y. and P.C.B. Phillips (1988), Statistical inference in regressions wiht integrated processes: Part 1, Econometric Theory, 4, 468-497.

Phillips, P.C.B (1986), Understanding spurious regressions in econometrics, Journal of Econometrics, 33, 311-340.

Phillips, P.C.B. (1987), Time series regression with a unit root, Econometrica, $55,277-301$.

Phillips, P.C.B. and S. Oularis (1990), Asymptotic properties of residual based tests for cointegration, Econometrica, 58, 165-193.

Phillips, P.C.B. and V. Solo (1992), Asymptotics for linear processes, Annals of Statistics, 20, 971-1001.

Pfann, G., Schotman, P., and Tschernig, R. (1996), Nonlinear interest rate dynamics and implications for the term structure, Journal of Econometrics $74,149-176$.

Saikkonen, P. (1991), Asymptotically efficient estimation of cointegration regressions, Econometric Theory, 7, 1-21.

Sargan, J.D. and A. Bhargava (1983), Testing residuals from least squares regression for being generated by the Gaussian random walk, Econometrica, 51, 153-174. 
Schmidt, P. and P.C.B. Phillips (1992), LM test for a unit root in the presence of deterministic trends, Oxford Bulletin of Economics and Statistics, $54,257-287$.

Stock, J.H. and M.W. Watson (1993), A simple estimator of cointegrating vectors in higher order integrated systems, Econometrica, 61, 783-820.

Wolters, J. (1995), On the term structure of interest rates - emprical results for Germany, Statistical Papers, 36, 193-214. 
Table 1: Critical Values

\begin{tabular}{|c|c|c|c|}
\hline$T$ & 0.10 & 0.05 & 0.01 \\
\hline$\kappa_{100}$ & 0.610 & 0.520 & 0.400 \\
$\kappa_{500}$ & 0.644 & 0.552 & 0.422 \\
$\xi_{100}$ & 0.059 & 0.042 & 0.024 \\
$\xi_{500}$ & 0.057 & 0.042 & 0.024 \\
\hline$\kappa_{100}^{*}$ & 0.400 & 0.370 & 0.320 \\
$\kappa_{500}^{*}$ & 0.394 & 0.364 & 0.316 \\
$\xi_{100}^{*}$ & 0.027 & 0.022 & 0.016 \\
$\xi_{500}^{*}$ & 0.023 & 0.019 & 0.013 \\
\hline$\widetilde{Q}_{100}[1]$ & -3.15 & -3.55 & -4.35 \\
$\widetilde{Q}_{500}[1]$ & -3.27 & -3.67 & -4.44 \\
$\widetilde{Q}_{100}[2]$ & -3.58 & -3.97 & -4.75 \\
$\widetilde{Q}_{500}[2]$ & -3.65 & -3.99 & -4.66 \\
$\widetilde{Q}_{100}[3]$ & -4.00 & -4.35 & -5.13 \\
$\widetilde{Q}_{500}[3]$ & -4.06 & -4.40 & -5.04 \\
$\widetilde{Q}_{100}[4]$ & -4.33 & -4.69 & -5.41 \\
$\widetilde{Q}_{500}[4]$ & -4.44 & -4.73 & -5.33 \\
\hline
\end{tabular}

Note: Critical values resulting from 10,000 replications of two independent random walk sequences. The statistic $\widetilde{Q}_{T}[k]$ represents a residual based ranked Dickey-Fuller $t$-test with $k$ regressors without a constant term. 
Table 2: Size and power $(\theta=0)$

\begin{tabular}{|c|c|c|c|c|c|c|}
\hline$f(x)=$ & \multicolumn{3}{|c|}{$x$} & $x^{3}$ & $\log (x)$ & $\tan \left(x_{t}\right)$ \\
\hline$\alpha$ & $\overline{\kappa_{T}}$ & $\overline{\xi_{T}}$ & CDF & $\mathrm{CDF}$ & $\mathrm{CDF}$ & $\mathrm{CDF}$ \\
\hline \multicolumn{7}{|c|}{ Size $(\beta=0)$} \\
\hline 1.00 & 0.049 & 0.049 & 0.050 & 0.098 & 0.051 & 0.077 \\
\hline \multicolumn{7}{|c|}{ Power $(\beta=0.5)$} \\
\hline 0.98 & 0.232 & 0.248 & 0.080 & 0.310 & 0.156 & 0.186 \\
\hline 0.95 & 0.288 & 0.332 & 0.225 & 0.491 & 0.229 & 0.295 \\
\hline 0.90 & 0.387 & 0.484 & 0.698 & 0.730 & 0.323 & 0.474 \\
\hline 0.80 & 0.551 & 0.708 & 0.999 & 0.866 & 0.410 & 0.616 \\
\hline \multicolumn{7}{|c|}{ Power $(\beta=1)$} \\
\hline 0.98 & 0.594 & 0.616 & 0.080 & 0.310 & 0.156 & 0.186 \\
\hline 0.95 & 0.733 & 0.792 & 0.225 & 0.491 & 0.229 & 0.295 \\
\hline 0.90 & 0.861 & 0.930 & 0.698 & 0.730 & 0.323 & 0.474 \\
\hline 0.80 & 0.953 & 0.993 & 0.999 & 0.866 & 0.410 & 0.616 \\
\hline
\end{tabular}

Note: Rejection frequencies resulting from 10,000 replications of the process given in (17). The sample size is $T=200$. Under the null hypothesis $(\alpha=1)$ we let $\beta=0$ and under the alternative $(|\alpha|<1), \beta=1$. The nominal size is 0.05 . The test statistic $\kappa_{T}$ and $\xi_{T}$ are defined in (7) and (8). CDF indicates a Dickey-Fuller $t$-test on the residuals of a cointegrating regression including a constant term.

Table 3: Power Against Threshold Cointegration

\begin{tabular}{|c|c|c|c|c|c|c|}
\hline & \multicolumn{3}{|c|}{$\lambda=0.5$} & \multicolumn{3}{c|}{$\lambda=1.5$} \\
\hline$a$ & $\kappa_{T}$ & $\xi_{T}$ & $\mathrm{CDF}$ & $\kappa_{T}$ & $\xi_{T}$ & $\mathrm{CDF}$ \\
\hline 0.98 & 0.600 & 0.629 & 0.081 & 0.547 & 0.571 & 0.083 \\
0.95 & 0.727 & 0.797 & 0.201 & 0.642 & 0.688 & 0.141 \\
0.90 & 0.854 & 0.929 & 0.655 & 0.783 & 0.840 & 0.327 \\
0.80 & 0.948 & 0.992 & 0.999 & 0.913 & 0.961 & 0.960 \\
0.60 & 0.989 & 1.000 & 1.000 & 0.974 & 0.998 & 1.000 \\
\hline
\end{tabular}

Note: Empirical powers computed from 10,000 replications of the threshold cointegrated process given in (17) and (18), where $T=200$. The parameter values are $\beta=1, \theta=0$ and $\sigma_{u}^{2}=1 /\left(1-a^{2}\right)$. The significance level is 0.05 . The test statistic $\kappa_{T}$ and $\xi_{T}$ are defined in (7) and (8). CDF indicates a Dickey-Fuller $t$-test on the residuals of a cointegrating regression including a constant term. 
Table 4: Testing correlated random walks

\begin{tabular}{|c|c|c|c|}
\hline$\rho$ & $\kappa_{T}^{*}$ & $\xi_{T}^{*}$ & $\widetilde{Q}_{T}[1]$ \\
\hline-0.9 & 0.123 & 0.010 & 0.004 \\
-0.6 & 0.065 & 0.017 & 0.013 \\
-0.4 & 0.051 & 0.026 & 0.023 \\
-0.2 & 0.050 & 0.036 & 0.034 \\
0.0 & 0.045 & 0.048 & 0.051 \\
0.2 & 0.048 & 0.062 & 0.068 \\
0.4 & 0.053 & 0.084 & 0.091 \\
0.6 & 0.064 & 0.114 & 0.124 \\
0.9 & 0.104 & 0.252 & 0.267 \\
\hline
\end{tabular}

Note: Empirical sizes resulting from 10,000 replications of the process given in (17) with $T=100$ and $\theta=0$ (no serial correlation). The nominal size is 0.05 . The parameter $\rho$ measures the correlation coefficient between the innovations of the random walks.

Table 5: Power against nonlinear cointegration relationships

\begin{tabular}{|c|c|c|c|c|c|c|}
\hline \multirow{2}{*}{$f(x)=$} & \multicolumn{2}{|c|}{$x^{3}$} & \multicolumn{2}{c|}{$\log (x)$} & \multicolumn{2}{c|}{$\tan (x)$} \\
\hline regressor: & $R_{T}\left(x_{t}\right)$ & $f\left(x_{t}\right)$ & $R_{T}\left(x_{t}\right)$ & $f\left(x_{t}\right)$ & $R_{T}\left(x_{t}\right)$ & $f\left(x_{t}\right)$ \\
\hline$\beta=0.01$ & 0.267 & 0.252 & 0.246 & 0.216 & 0.237 & 0.226 \\
$\beta=0.05$ & 0.473 & 0.485 & 0.701 & 0.676 & 0.549 & 0.548 \\
$\beta=0.1$ & 0.714 & 0.746 & 0.957 & 0.955 & 0.834 & 0.855 \\
$\beta=0.5$ & 0.974 & 0.988 & 1.000 & 1.000 & 0.999 & 1.000 \\
\hline
\end{tabular}

Note: Simulated power from a score tests using $R_{T}\left(x_{t}\right)$ and $f\left(x_{t}\right)$ as additional regressors. The sample size is $T=200$. 
Table 6: The cointegration relationship with R1

\begin{tabular}{|l|c|c|c|c|c|c|}
\hline Regr. & CDF & $\widetilde{Q}_{T}[1]$ & $\kappa_{T}^{*}$ & $\xi_{T}^{*}$ & nonlin & corr \\
\hline R2 & $-4.138^{*}$ & $-5.246^{*}$ & $0.347^{*}$ & $0.010^{*}$ & 0.003 & 0.935 \\
R3 & $-3.591^{*}$ & $-4.362^{*}$ & $0.357^{*}$ & $0.014^{*}$ & 0.012 & 0.873 \\
R4 & $-3.373^{*}$ & $-4.083^{*}$ & $0.366^{*}$ & $0.015^{*}$ & 0.034 & 0.817 \\
R5 & -3.213 & $-3.829^{*}$ & $0.380^{*}$ & $0.017^{*}$ & 0.107 & 0.774 \\
R10 & -2.702 & -3.216 & 0.471 & 0.024 & 0.489 & 0.675 \\
\hline
\end{tabular}

Note: "CDF" denotes the Dickey-Fuller $t$-test applied to the residuals of the cointegration regression, $\widetilde{Q}_{T}[1]$ is the same test applied to the ranks, $Z_{T}(m)$ is given in (12), "nonlin" indicates the test for nonlinearity using the ranks as additional regressors, "corr" denotes the mutual correlation between the differenced series. "*" indicates significance at the 0.05 significance level. 